\title{
Mobile Reminder System for Furthering Patient Adherence Utilizing Commodity Smartwatch and Android devices
}

\author{
Ilias Maglogiannis \\ Department of Digital Systems \\ University of Piraeus \\ Piraeus, Greece \\ imaglo@unipi.gr
}

\author{
George Spyroglou, \\ Greek Research and Education Network \\ (GR-NET) \\ Athens, Greece
}

\author{
Christos Panagopoulos, Maria \\ Mazonaki, Panayiotis Tsanakas \\ School of Electrical and Computer \\ Engineering \\ National Technical University of Athens \\ Athens, Greece
}

\begin{abstract}
Patients' non-adherence is a common problem that significantly reduces the effectiveness of treatments and increases mortality and healthcare costs. Forgetfulness is reported as a usual barrier to adherence. Electronic reminder systems consist an increasingly used type of intervention, which assists patients that are facing this problem. Important requirements regarding the design of such systems are multimodality, personalization and easy integration into the patient's daily life. The goal of this work is to present a multimodal electronic reminder system that supports the use of smart devices and utilizes the recently introduced Pebble smartwatch. The system employs cloud technology and also allows for other types of users with a supportive role in the patient's health care, such as the patient's doctor and pharmacist. Patient compliance is measured automatically with the method of self-reporting. The paper includes a brief evaluation of the user interface and some future research directions.
\end{abstract}

Keywords-electronic reminders; smartwatch; medication adherence; patient compliance; multimodal reminder system; assisted living; home care

\section{INTRODUCTION}

Adherence can be defined as the extent to which a patient acts in accordance to medical or health advice. Adherence is important for the success of a treatment, especially in the case of chronic illnesses. It is associated with lower mortality and can be indicative of an overall healthy behavior (healthy adherer effect) [1]. Patients' non-adherence is a persistent problem that undermines treatment benefits and increases healthcare costs [2-3]. In the 2003 WHO report on medication adherence, it is stated that approximately $50 \%$ of patients with chronic illnesses do not take medications as prescribed [2]. There are several barriers to adherence, including concerns about efficacy or side effects, inconvenience, poor doctorpatient relationship and lack of motivation [4].

Adherence can be measured directly (i.e. detection of chemicals in body fluids, direct observation of the patient) or, more commonly, indirectly (i.e. medication monitoring, selfreports). Direct measures are generally considered more reliable, but they are also costly, labor-intensive and usually not possible [5]. Medication monitoring usually involves the measurement of how many pills a patient has taken with accuracy, but provides no information about the time of intake. Self-reports (e.g. diaries, questionnaires), although subject to bias, are a very common and easy to implement methodology for the measurement of patient adherence.

According to experts, simple interventions that are easy for both professionals and patients and incorporated in a daily practice are the most promising approaches in furthering patients' adherence [6]. As forgetfulness is one of the most common barriers to adherence, a simple intervention in the form of electronic reminders can be an effective solution for patients who are unintentionally non-adherent [7]. Electronic reminder systems can be an important feature of assisted living applications [8]. Many users of such applications have problems remembering information and important tasks. Moreover, considering that the primary users of home care technology are usually elderly or people with significant sensory impairments, multimodality is a necessary feature for reminder systems [8].

In this paper we propose a multimodal reminder system, as part of an assisted living application. The system exploits an Android device and a smartwatch that communicate via Bluetooth interface. The Pebble smartwatch was selected for the prototype implementation due to its several positive characteristics [9]. The main functionality of the system involves the creation of reminders using a PC or their Android device, which are stored in a Cloud infrastructure. For every reminder, the patient is notified at the chosen time, with audio and visual alerts. In addition, reminder notifications are pushed to the smartwatch. Other registered users, such as the patient's physician or pharmacist, can use a web application and create or update reminders. The system can also work in conjunction with e-prescribing (e-Rx) systems to automatically create reminders regarding medication intake [12]. The overall architecture of the proposed system is illustrated in Fig. 1.

The rest of the paper is organized as follows: In Section II, background information on electronic reminder applications and the Pebble smartwatch is provided. Next, in Section III the proposed system is presented in detail, while Sections IV and V are devoted to a brief evaluation of the proposed system, concluding remarks and some future research directions. 


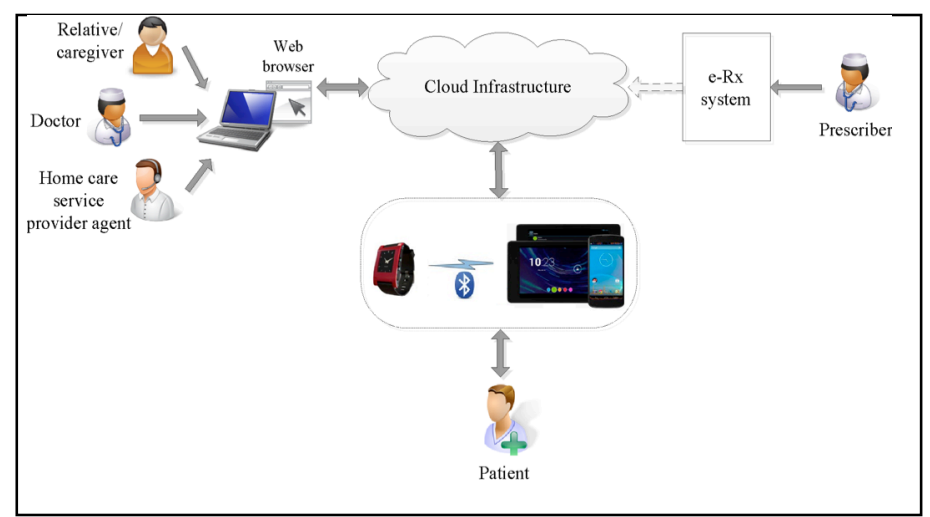

Figure 1. Architecture of the proposed electronic reminder system.

\section{BACKGROUND INFORMATION}

\section{A. Electronic Reminder Applications}

Electronic reminders are nowadays increasingly being used as an effort to improve drug adherence. This practice is based on the principles of behavioral learning theory [7]. Intervention with electronic reminders can be implemented in a variety of ways, including short message service (SMS), electronic reminder devices and text messages sent to the patient's pager. Studies of such systems have provided evidence of short-term effectiveness of this type of intervention [7]. Solutions that have been effective for longterm treatments, however, usually combine different kinds of interventions, including counseling, telephone follow-ups and supportive care $[7,10]$. In the case the electronic reminder system is integrated in a home care application, additional mechanisms can be set in motion when reminders are insufficient in ensuring patient compliance (e.g. automatic notification of the patient's doctor for a follow-up call).

A comprehensive study of the requirements of home reminder systems exists in [8]. According to the study, modality preferences varied between different groups of people, so it is important for a reminder system to be configurable. Device preference also varied, with popular choices including mobile phones, TVs, watches or screens placed in specific rooms. Other important parameters are seamless integration into the life and home of the patient, and privacy in shared spaces.

The system proposed in this work aims to integrate seamlessly into the patient's daily life, by utilizing smart and wearable devices. The Android platform enables the system to run on a variety of devices, including smartphones, tablets or TVs. The research work presented here focuses on smartwatches, which are a recently introduced class of smart devices in human centered computing. Since smartwatch displays are relatively small, reading text notifications can be a challenge for the elderly. In this case, smartwatch notifications are used as generic reminders, which are often preferred by patients [8]. The capabilities of the Pebble smartwatch, which is employed in the proposed system, are presented in the next section.

\section{B. The Pebble Smartwatch}

The Pebble is a popular state of the art smartwatch, which was released in 2013, by Pebble Technology Corporation. It can communicate with Android and iOS devices using the Bluetooth 4.0 (Low Energy) protocol and receive vibrating alerts for text messages, incoming calls or social media notifications. It features a 1.26 -inch $144 \times 168$ pixel, black and white e-paper display with a backlight, a vibrating motor, integrated sensors, such as an accelerometer, and a rechargeable battery that lasts for 5-7 days [9]. An open Pebble software development kit (SDK) is available to developers. Applications for the Pebble are distributed through the Pebble appstore.

\section{THE PROPOSED SYSTEM}

The proposed system is a cloud-based service that consists of three software modules. The first module is a web-based application, which enables registered users to create reminders and monitor patient adherence. The second module is an Android application, which is used by the patient for managing reminders. The application has an integrated background service that activates notifications and communicates with the smartwatch. The third module runs on the Pebble and handles notifications received from the Android device.

\section{A. The Android Application}

The user can create reminders using the Android application entering as inputs a description and the desired date and time of notification. Each reminder has also a status. The status is set as "Forthcoming" upon creation, changes to "Pending" upon notification and lastly, the user can mark a reminder as "Completed", when the associated task is accomplished. In order to avoid drug intake overlap, reminder notifications expire (cease) after a preset time limit. The user can specify the duration of the alarm or choose the default setting, which is 1 hour. If the associated task is recurring, the user has the option to set a recurrence interval (i.e. daily, weekly, monthly or yearly), in which case copies of the reminder will be automatically created when the previous reminder is completed or as soon as it expires. Reminders can

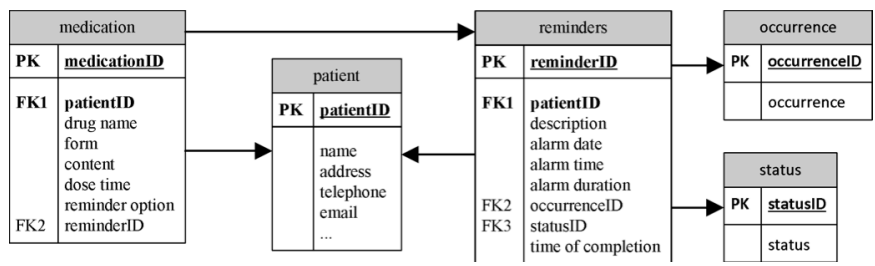

Figure 2. Part of the database schema.

also be created automatically, for such tasks as medication intake in conjunction with other parts of the overall home care system (e.g. electronic records of prescribed medication regimens). The part of the database schema, which relates to the reminder application is illustrated in Fig. 2. 
The integrated background service connects to the cloud and calls a web service every 60 seconds, in order to fetch all non-completed reminders, with the patient's ID, that are set for the current date. Fetched reminders are then filtered by the service, keeping only pending reminders that have not expired and forthcoming reminders with notification time set within the next 3 minutes. If such reminders are found, the service activates an audio alert and displays the respective descriptions on the screen, along with buttons for marking the reminder as "Completed" or dismissing the notification (Fig. 3 ). The service sends a message to the server to change the status of the reminder to "Completed" or "Pending" respectively. If the user presses "Later", the alert stops and the user will be notified again at the next call.

Meanwhile, if the Pebble application is running as well and a connection has been established between the two devices, the service pushes a notification to the Pebble. In case of loss of Internet connection, the service automatically reconnects to the server as soon as connection to the Internet is established again.

\section{B. The Pebble Application}

The main screen of the Pebble application consists of a minimal clock. The application checks whether an active Bluetooth connection with the Android device is established and displays the connection status at the bottom of the main screen (Fig. 4). Whenever the devices is disconnected the system automatically triggers a reconnection and, if connection is not established again within a few seconds, the Pebble application notifies the user with a vibration.

When a notification is pushed to the Pebble, the proper event handler turns the screen backlight on, changes the displayed layer, from the main screen to notification screen, and activates the vibration motor. The user can dismiss the notification by pressing one of the watch's buttons, in which case an appropriate string value is sent to the Android device to dismiss the reminder and the Pebble application returns to its previous state.

The connection of the two devices is managed through the local Pebble service, which is part of the Official Pebble Android app. If, for any reason, the connection between the two devices is lost, the Pebble service automatically attempts to reconnect. Communication between the devices is achieved using the AppMessage API. Messages are exchanged as

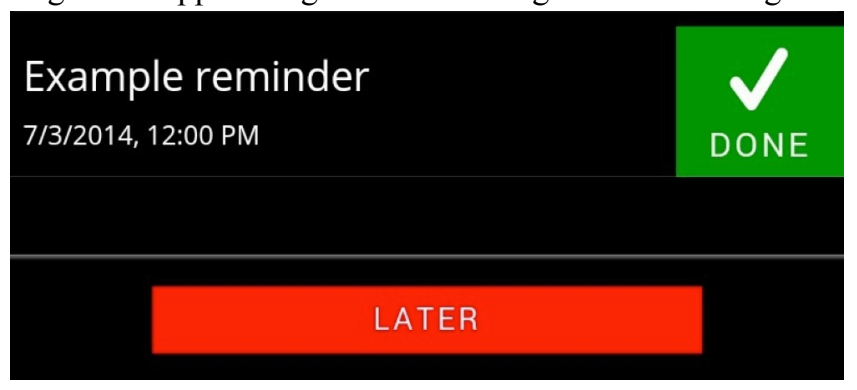

Figure 3. Reminder notification on the Android device.

arbitrary sets of key/value pairs, which are stored in the form of a Dictionary. Every message needs to get either "acknowledged" or "not acknowledged" by the receiving
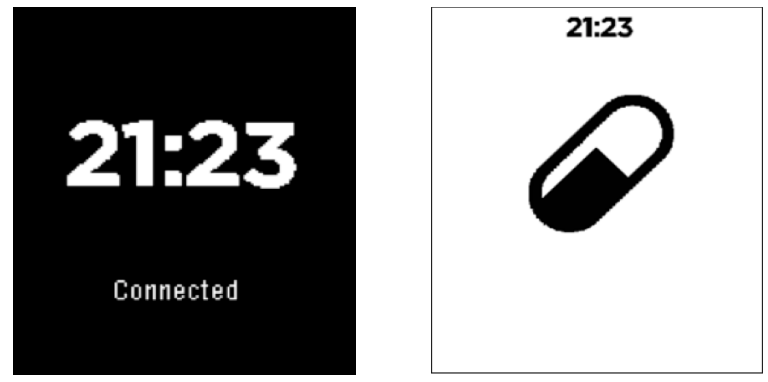

Figure 4. Screenshots of the Pebble application, in case of an idle connection and in case of a reminder alert.

device. If not, it will result in a time-out failure. On the Pebble side, this is handled by the AppMessage subsystem implicitly.

\section{The Web Application}

The system supports registration of other users that are involved in the patient's health care, including doctors, pharmacists and close family. Each user can login to the system and use the web application to create reminders for the associated patient. Each type of user views a different version of the web application with specific permissions depending on their identity (e.g. the patient's doctor can also edit prescribed medication records).

\section{Web Technologies Used in the Implementation}

In the development of the system, Apache Tomcat was used for the web server, MySQL for the database server and jPlaton for the application server, which is an integrated environment based on Java and XML. Applications built on jPlaton are independent from operating systems, databases and system architectures and may also run on a web-based environment. Server side development was done with the PlatonScript scripting language. More details about the jPlaton and PlatonScript technologies can be found at the official website [11].

On the client side, HTML, CSS and JavaScript were used for development. The use of JavaScript allows for an interactive and visually appealing web application, compatible with most of the major web browsers, and provides fast processing for the end user. Most importantly, development with JavaScript reduces the strain on the web server, thus giving the system scalability.

\section{An Evaluation of The System}

\section{A. Evaluation of the User Interface}

The presented system meets many of the design requirements mentioned in section II. The selection of the Android platform allows the use of various different devices, most of which are widely available and used by patients on a daily basis. The need for multimodality is covered, by using audio, visual and vibration alerts. In addition, privacy in shared spaces can be achieved easily, by silencing the Android device, as notifications on the Pebble are discreet. The system is very easy to use, as it requires minimum user input.

Updates of the official Pebble app for Android have fixed most of the original issues with Bluetooth connection and 
pairing of the smartwatch with the Android device, while automatic reconnections after communication loss improve the reliability of the system. An important limitation of the system, however, is that it cannot operate without an active Internet connection, since reminders are not locally stored.

\section{B. Measuring Patient Compliance}

The marking of a reminder as "Completed" acts as a form of self-report by the patient, which can be used by the system to create compliance reports. An advantage of this method is that it is unobtrusive. Moreover, the patient reports are automatically updated for every medication intake, thus avoiding potential bias or errors of other self-report methods.

The rate of compliance is expressed as the proportion of medication reminders that were completed over the total number of medication reminders (CR/TR), implying number of pills taken over the number of pills prescribed. Compliance rates can be drug specific or measured over a period of time. Another important measurement is the mean response time (MRT) to medication reminders that were marked as "Completed", as proper adherence to a medication regimen requires medication intake in specific time intervals. Response time (RT) is the interval between the initial time of notification for a specific reminder and the time of completion.

An exemplary usage scenario over four days is described in Table I. In this scenario, the patient is an elderly person suffering from mild dementia. There are two prescribed drugs, A and B. Drug A has to be taken twice daily and drug B once daily, with a total of three reminders per day. Table II describes the corresponding report for this scenario.

In case of consecutive failed reminders, the system automatically sends an appropriate alert, via SMS or email, to a predefined list of persons, which can include the patient's doctor or a relative. As such, an alert would be sent on day 4 of this scenario. Alerts are also sent by the system in case the patient fails to take consecutive doses of a drug, as in day 3 of the scenario.

TABLE I. USAGE SCENARIO RESPONSE TIMES

\begin{tabular}{|c|c|c|c|}
\hline \multirow[b]{2}{*}{ Days } & \multicolumn{3}{|c|}{ Response times } \\
\hline & $\begin{array}{c}1^{\text {st }} \text { reminder } \\
\text { (drug } A)\end{array}$ & $\begin{array}{c}2^{2^{n d}} \text { reminder } \\
\text { (drug } B)\end{array}$ & $\begin{array}{c}3^{\text {rd }} \text { reminder } \\
\text { (drug } A)\end{array}$ \\
\hline Day 1 & $0 \mathrm{~min}$ & $0 \mathrm{~min}$ & $0 \mathrm{~min}$ \\
\hline Day 2 & $30 \mathrm{~min}$ & Missed & $60 \mathrm{~min}$ \\
\hline Day 3 & Missed & $30 \mathrm{~min}$ & Missed \\
\hline Day 4 & $0 \mathrm{~min}$ & Missed & Missed \\
\hline
\end{tabular}

TABLE II. USAGE SCENARIO REPORT

\begin{tabular}{|c|c|c|}
\hline Drug & $\boldsymbol{C R} / \boldsymbol{C T}$ & $\boldsymbol{M R T}$ \\
\hline Drug A & $62.5 \%$ & $18 \mathrm{~min}$ \\
\hline Drug B & $50 \%$ & $15 \mathrm{~min}$ \\
\hline \hline Total & $58.3 \%$ & $17 \mathrm{~min}$ \\
\hline
\end{tabular}

\section{CONCLUTIONS AND FUtURE RESEARCH DiRECTIONS}

This paper presents a user-friendly, multimodal and customizable electronic reminders system. The proposed system has been designed to meet many essential requirements of reminder systems that are used as part of home care applications. An important advantage is the utilization of lowcost, widely available devices. The system also provides an easy and automated method of measuring patient nonadherence by self-reports. A study of the system in practice shall be conducted in order to verify expected results in patient adherence and test the reliability of the system's adherence reports. In addition, future work can focus on furthering the system's customizability and autonomy. The Android app can also be adjusted to store reminders and user-interaction data locally, so that the system can operate even without an active Internet connection.

\section{ACKNOWLEDGMENT}

Part of the work reported here has been carried out in the framework of national project Providing Integrated eHealth Services for Personalized Medicine utilizing Cloud Infrastructure (PinCloud), led by the University of Piraeus, conducted in the context of the National Strategic Reference Framework NSRF 2007-2013, Cooperation 2011 and cofunded by the European Commission.

\section{REFERENCES}

[1] S. H. Simpson, "A meta-analysis of the association between adherence to drug therapy and mortality," BMJ, vol. 333, no. 7557, pp. 15-0, Jul. 2006.

[2] E. SabateÏ and World Health Organization, Adherence to long-term therapies evidence for action. Geneva: World Health Organization, 2003

[3] M. C. Sokol, K. A. McGuigan, R. R. Verbrugge, and R. S. Epstein, "Impact of medication adherence on hospitalization risk and healthcare cost," Medical care, vol. 43, no. 6, pp. 521-530, 2005.

[4] C. West, Fenerty, Feldman, Kaplan, and Davis, "The effect of reminder systems on patients' adherence to treatment," Patient Preference and Adherence, p. 127, Feb. 2012.

[5] K. C. Farmer, "Methods for measuring and monitoring medication regimen adherence in clinical trials and clinical practice," Clinical Therapeutics, vol. 21, no. 6, pp. 1074-1090, 1999.

[6] S. van Dulmen, E. Sluijs, L. van Dijk, D. de Ridder, R. Heerdink, and J. Bensing, "Furthering patient adherence: A position paper of the international expert forum on patient adherence based on an Internet forum discussion," BMC Health Services Research, vol. 8, no. 1, p. 47, 2008.

[7] M. Vervloet, A. J. Linn, J. C. M. van Weert, D. H. de Bakker, M. L. Bouvy, and L. van Dijk, "The effectiveness of interventions using electronic reminders to improve adherence to chronic medication: a systematic review of the literature," Journal of the American Medical Informatics Association, vol. 19, no. 5, pp. 696-704, Sep. 2012.

[8] M. R. McGee-Lennon, M. K. Wolters, and S. Brewster, "User-centred multimodal reminders for assistive living," in Proceedings of the SIGCHI Conference on Human Factors in Computing Systems, 2011, pp. 2105-211.

[9] Official Pebble Web Site https://getpebble.com.

[10] R. B. H. Haynes, E. Ackloo, N. Sahota, H. P. McDonald, and X. Yao, "Interventions for enhancing medication adherence," Cochrane Database of Systematic Reviews, no. 2, 2008.

[11] Official jPlaton Web Site http://www.b-open.gr/index.php/en/jplatontechnology

[12] Doukas, C., Maglogiannis, I., Tsanakas, P., Malamateniou, F., Vassilacopoulos, G. mPharmacy: A system enabling prescription and personal assistive medication management on mobile devices (2011) Lecture Notes of the Institute for Computer Sciences, Social-Informatics and Telecommunications Engineering, 55 LNICST, pp. 153-159. 